\title{
SEPTICAEMIC MELIOIDOSIS COMPLICATED BY SEPTIC ARTHRITIS IN A BANGLADESHI MALE: A CASE REPORT
}

\author{
HASNA FAHMIMA HAQUE ${ }^{1}$, SUCHISMITA DEBNATH ${ }^{2}$, SAMIRA RAHAT AFROZE ${ }^{3}$, FARHANA AFROZ ${ }^{3}$, \\ MUHAMMAD ABDUR RAHIM ${ }^{4}$, AKM MUSA ${ }^{5}$
}

\begin{abstract}
Melioidosis is an uncommon disease in Bangladesh but in recent years increasing numbers of cases are being diagnosed. A case of septicaemic melioidosis occurring in a young Bangladeshi returning worker is reported here. The patient presented with fever and features of septic arthritis. Diagnostic work-up revealed the growth of Burkholderia pseudomallei from blood and synovial fluid cultures. This case highlights the importance of high index of clinical suspicion for melioidosis in appropriate clinical scenario.
\end{abstract}

Key words: Bangladesh, Burkholderia pseudomallei, fever, melioidosis, septic arthritis.

Received: 02 June 2018

Accepted: 10 June 2018

DOI: http://dx.doi.org/ 10.3329/ bjmed.v29i2.37946

\section{Introduction}

Melioidosis is an infectious disease caused by infection with Burkholderia pseudomallei, a Gramnegative bacterium found in soil and surface water. Patients with diabetes mellitus are at increased risk for infection by B. pseudomallei. Patients may present with fever, pneumonia, cutaneous and organ abscesses, septic arthritis, genito-urinary infections, etc. Melioidosis is an uncommon diagnosis in Bangladesh ${ }^{1}$ though nearly 17,000 cases are estimated to occur annually. ${ }^{2}$ Here, we report a case of melioidosis occurring in a diabetic patient who presented with fever and septic arthritis.

\section{Case Report}

A 36-year-old Bangladeshi man presented with two and half months history of high grade continued fever along with pain and swelling involving the right shoulder and bilateral knee joints. He returned to Bangladesh two months ago from Malaysia, where he had been involved in farming for few years. His symptoms started while staying in Malaysia and because of worsening symptoms he came back home. For his problems, he initially consulted at Gopalgonj, his local district in Bangladesh and two weeks previously he received an intra-articular steroid injection in right shoulder joint resulting in no benefit. His fever and joint symptoms continued to worsen and he developed vomiting, several episodes a day, for few days before admission under our care. His medical history included diabetes mellitus for which he had been taking metformin.

On admission, the patient was looking very ill, he was febrile with a temperature of $102^{\circ} \mathrm{F}$, mildly icteric, tachycardic (pulse 110/min) but had normal blood pressure (125/70 mm Hg). His right shoulder joint (Figure 1) and knee joints (Figure 2) were swollen, hot and tender. He had 2-cm splenomegaly below the

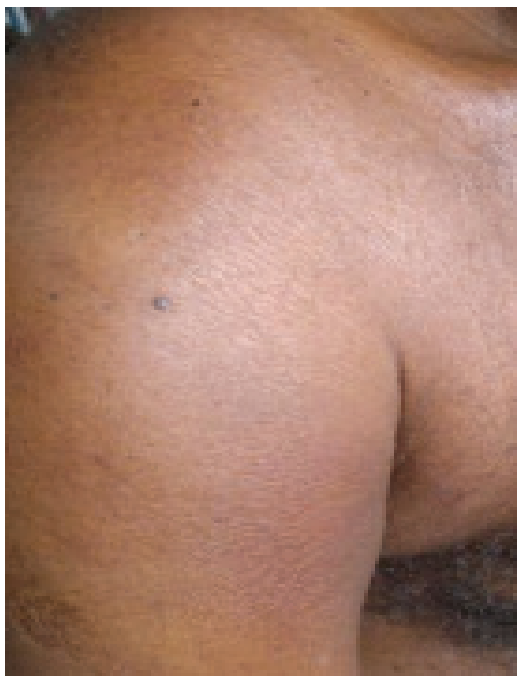

Fig.-1: Swelling of right shoulder joint

1. Assistant Professor, Department of Internal Medicine, BIRDEM General Hospital, Dhaka, Bangladesh.

2. RMO, Department of Internal Medicine, BIRDEM General Hospital, Dhaka, Bangladesh.

3. Registrar, Department of Internal Medicine, BIRDEM General Hospital, Dhaka, Bangladesh.

4. Assistant Professor, Department of Nephrology, BIRDEM General Hospital, Dhaka, Bangladesh.

5. Professor and Head, Department of Internal Medicine, BIRDEM General Hospital, Dhaka, Bangladesh.

Address of Correspondence: Dr. Muhammad Abdur Rahim, Assistant Professor, Department of Nephrology, BIRDEM General Hospital, Dhaka, Bangladesh. email: muradrahim23@yahoo.com 


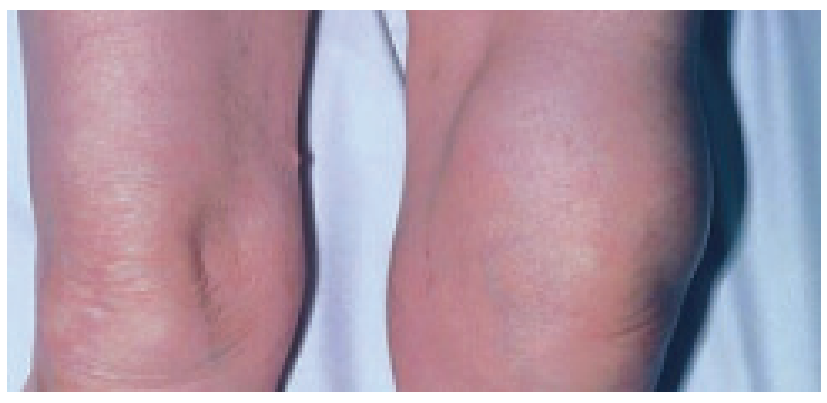

Figure 2. Swelling of knee joints more on left

left sub-costal margin and bilateral non-proliferative diabetic retinopathy. Bedside urine showed glycosuria $(+)$ and proteinuria $(+)$.

He had anaemia (haemoglobin $8.6 \mathrm{gm} / \mathrm{dL}$ ), neutrophil leukocytosis (total white cells $31,000 / \mathrm{mm}^{3}$, neutrophil $84.9 \%$ ) and high erythrocyte sedimentation rate $\left(97 \mathrm{~mm}\right.$ in $1^{\text {st }}$ hour). Liver biochemistry was altered (bilirubin $6 \mathrm{mg} / \mathrm{dL}$, alanine aminotransferase $33 \mathrm{U} / \mathrm{L}$, aspartate aminotransferase $50 \mathrm{U} / \mathrm{L}$ and alkaline phosphatase $530 \mathrm{U} / \mathrm{L}$, serum total protein 65. $2 \mathrm{gm} / \mathrm{dL}$ and albumin $18.2 \mathrm{gm} / \mathrm{dL}$ ) and there was hyponatraemia (serum sodium $123 \mathrm{mmol} / \mathrm{L}$ ). Random blood glucose was $21.5 \mathrm{mmol} / \mathrm{L}$ and glycated haemoglobin (HbA1c) was $8.1 \%$. Abdominal ultrasonography revealed mild hepato-splenomegaly with coarse hepatic parenchyma. Upper gastrointestinal tract endoscopy showed congestive gastropathy and he had serological evidence of hepatitis $B$ virus infection (positive total anti-HBc). Blood culture revealed growth of B. pseudomallei as was true for synovial fluid culture (from left knee joint).

He was initially started with ceftazidime $1 \mathrm{gm}$ intravenously every 8 hours, which was converted to 2 gm intravenously every 8 hours after receiving the blood and synovial fluid culture reports and continued for 4 weeks. He was discharged in afebrile state with oral co-trimoxazole and doxycycline with the plan to continue for 22 weeks. He is on regular follow-up with us and improving satisfactorily.

\section{Discussion}

Melioidosis is endemic in south-east Asia and northern Australia, but imported cases have been reported elsewhere. ${ }^{2}$ Isolation of the causative organism, B. pseudomallei, from soil specimen ${ }^{3}$, significant sero-epidemiological survey reports ${ }^{3,4}$ and autochthonous cases ${ }^{1,5,6}$ had confirmed Bangladesh as endemic for melioidosis.

Common presentations of melioidosis include fever, pneumonia, abscesses, septic arthritis and genitourinary infections. ${ }^{5,6}$ Clinical presentation of melioidosis often mimics tuberculosis and in tuberculosis endemic countries like Bnagladesh, it is not unlikely that cases are started with empiric anti-tuberculosis therapy. ${ }^{6,7}$ Septic arthritis is common and generally complicates septicaemic melioidosis. ${ }^{8,9}$ Patients may develop metastatic abscess even while on antibiotic treatment.

Diabetes mellitus is an established risk factor for melioidosis and occasionally an infective episode by B. pseudomallei may unmask undetected diabetes. 5,6 Other risk factors include alcoholism, steroid intake ${ }^{5}$, renal failure, chronic liver disease, thalassaemia, chemotherapy etc. ${ }^{1}$ Our patient had diabetes and hepatitis B virus associated chronic liver disease. Occupational risk factors for melioidosis include farming specially working in wet paddy fields and recreational exposures to soil and waters. The bacterium enters through an abraded skin or by inhalation. ${ }^{5}$

Like any other infectious diseases, culture remains the gold standard for diagnosis of melioidosis. $B$. pseudomallei readily grows in ordinary media from representative samples like blood, urine and pus. Important is to have a high index of clinical suspicion and a good liaison between the treating physician and the microbiologists. ${ }^{1,5}$ Growth of Pseudomonaslike organisms which are resistant to aminoglycosides and polymyxin/colistin and sensitive to co-amoxiclav is important clue for laboratory personnel and microbiological diagnosis. ${ }^{5}$

Treatment of melioidosis consists of initial intensive therapy with ceftazidime or carbapenems for several weeks followed by eradication phase with oral antibiotics for months often in combinations. ${ }^{5}$ Duration of antibiotic treatment depends on severity of disease and sites of involvement. Joint and bone diseases require prolonged treatment and often merits drainage. ${ }^{8}$

Question may arise, whether the index patient acquired melioidosis from Malaysia or in Bangladesh? Such issue has recently been asked by researchers ${ }^{10}$ and the answer is not straight forward. But, as the symptoms of our patient started while in Malaysia, he might have acquired infection in Malaysia. Molecular techniques may answer the question.

\section{Conclusion}

Melioidosis is an uncommon disease in Bangladesh. Cases are increasingly being diagnosed now-a-days, primarily because of improving awareness regarding existence of melioidosis in Bangladesh, among physicians. We further emphasize physicians to have a high index of clinical suspicion in appropriate 
clinical contexts and every septic arthritis cases should have blood and synovial fluid cultures before starting antibiotics.

\section{References}

1. Rahim MA, Afroze SR, Barai L, Uddin KN. Melioidosis: Truly Uncommon or Uncommonly Diagnosed in Bangladesh? A Case Report. Birdem Med J 2015; 5 (Supplement 1): 49-51.

2. Limmathurotsakul D, Golding N, Dance DA, Messina JP, Pigott DM, Moyes CL, et al. Predicted global distribution of Burkholderia pseudomallei and burden of melioidosis. Nat Microbiol 2016; 1(1). pii: 15008.

3. JilaniMSA, Robayet JAM, Mohiuddin M, Hasan MR, Ahsan CR, Haq JA. Burkholderia pseudomallei: Its Detection in Soil and Seroprevalence in Bangladesh. PLOS Neglected Tropical Diseases 2016; 10(1): e0004301.

4. Maude RR, Maude RJ, Ghose A, Amin MR, Islam MB, Ali M, et al. Seroepidemiological surveillance of Burkholderia pseudomallei in Bangladesh. Transactions of the Royal Society of Tropical Medicine and Hygiene 2012; 106(9): 576-578.

5. Rahim MA, Samad T, Ananna MA, Haque WMM. Genitourinary melioidosis in a Bangladeshi farmer with IgA nephropathy complicated by steroidinduced diabetes mellitus. Saudi J Kidney Dis Transpl 2018;29(3):709-713.

6. Afroze SR, Barai L, Rahim MA, Haque HF, Afroz F, HoqueMT, et al. Socio-demographic, Clinical and Laboratory Characteristics of Melioidosis: Four-Year Experience of Managing Consecutive 11 Cases in a Tertiary Care Hospital of Bangladesh. BIRDEM Med J 2017; 7(1): 28-37.

7. Chow TK, Eu LC, Chin KF, Ong KC, Pailoor J, Vadivelu J, et al. Case Report: Incidental Splenic Granuloma due to Burkholderia pseudomallei: A Case of Asymptomatic Latent Melioidosis? Am J Trop Med Hyg 2016; 94(3): 522-524.

8. Raja NS, Scarsbrook C. Burkholderia pseudomallei Causing Bone and Joint Infections: A Clinical Update. Infect Dis Ther 2016; 5(1): 17-29.

9. Afroze SR, Rahim MA, Barai L, Uddin KN. Disseminated melioidosis involving skin and joint: a case report. Ibrahim Med Coll J 2015; 9(2): 55-57.

10. Rahim MA, Afroze SR, Afroz F, Haque HF, Barai L, Ahmed JU, et al. Melioidosis among returning workers in Bangladesh: imported or endemic? Sri Lankan Journal of Infectious Diseases 2017; 7 (Supplement S27):34-35. 